\title{
Chandra X-ray Observations of Dwarf Starburst Galaxies
}

\author{
Jürgen Ott \\ CSIRO Australia Telescope National Facility, Cnr Vimiera \& Pembroke \\ Rds, Marsfield NSW 2122, Australia \\ Fabian Walter \\ National Radio Astronomy Observatory, P.O. Box O, Socorro, NM \\ 87801, USA \\ Elias Brinks \\ Instituto Nacional de Astrofísica, Optica y Electrónica (INAOE), \\ Puebla, Mexico \\ Ulrich Klein \\ Radioastronomisches Institut, Universität Bonn, Auf dem Hügel 71, \\ 53121 Bonn, Germany
}

\begin{abstract}
We obtained X-ray observations for a sample of eight nearby dwarf starburst galaxies from the Chandra X-ray Observatory. Five galaxies of our sample show extended (size: $1-10 \mathrm{kpc}$ ), diffuse X-ray emission which can be attributed to a hot thermal plasma. This phase of the interstellar medium purportedly drives the expansion of supergiant shells. A comparison of the derived gas parameters with theoretical models reveals that the hot gas in principle is capable to escape from the gravitational potential of the host galaxy. However, the outflows appear to be contained in those cases where an extended envelope or massive tidal features of neutral gas exist.
\end{abstract}

\section{Introduction}

The high star formation rate in starburst galaxies is responsible for the deposition of a large amount of mechanical energy into the interstellar medium (ISM). The energy is ejected by strong stellar winds and multiple type II supernova (SN) explosions. In the course of this process, the ISM is heated to coronal temperatures and galactic winds develop along the direction of the steepest gradient in the ISM, typically along the minor axis of the host galaxy (see e.g, the review by Heckman 2002 and references therein). Theoretical models (e.g., Mac Low \& Ferrara 1999; Ferrara \& Tolstoy 2000) predict that for a given mechanical starburst luminosity the mass of the host galaxy is an important factor which determines the fate of the ejected gas. For a large mass $\left(z 10^{8-9} \mathrm{M}_{\odot}\right)$ the gas will be retained by the galaxy and the hot component will fall back on the disk after cooling down in the halo. For lower masses, part of the metal-enriched 
material is able to escape from the gravitational potential of its host. At the low mass end $\left(\lesssim 10^{6} \mathrm{M}_{\odot}\right)$, all of the ISM within the galaxy is lost to the intergalactic (or intragroup) medium (IGM).

Low mass galaxies, i.e., dwarf galaxies are the most numerous type of galaxy in the local universe. Looking back in time, theories of cold dark matter predict hierarchical structure formation. Consequently, dwarf galaxies must have been even more numerous at higher redshifts. Starbursts developing in dwarf galaxies therefore may be an important mechanism to enrich the IGM with metals at all epochs. In addition, dwarf galaxies, in general, exhibit a somewhat lower metallicity of $\sim 1 / 10$ solar. This is an indication that they might be similar to objects at larger lookback times and/or might have lost a large portion of freshly produced metals by galactic winds.

$\mathrm{X}$-ray observations are very well suited for the investigation of starbursts as they are the only means by which to detect the very hot phase $\left(\sim 10^{6-7} \mathrm{~K}\right)$ of a galactic wind which is the first thermalized phase of the deposited, mechanical energy. Metals ejected from supernovae enrich this hot, coronal phase.

\section{Sample Selection and Observations}

To study the hot phase of the ISM in starburst dwarf galaxies we selected a sample of eight objects observed with the Chandra X-ray observatory (ACIS-S3). All of the galaxies were previously detected in $\mathrm{X}$-ray emission by former missions, in particular with ROSAT. The unprecedented high angular resolution and the large collecting area of Chandra, however, allows much more sensitive observations and the identification (and removal) of point sources from any diffuse component. Our sample consists of the following galaxies: I Zw 18, VII Zw 403, IC 2574, NGC 3077, NGC 4214, NGC 4449, NGC 5253, and He 2-10. Note that IC 2574 is not a starburst galaxy in a strict sense. It was added to the sample as it contains a strong star forming region in a region to the north. Even though a detailed analysis of the Chandra data has been performed in the case of NGC 3077 (Ott, Martin, \& Walter 2003), NGC 1569 (Martin, Kobulnicky, \& Heckman 2002), NGC 4449 (Summers et al. 2003), and IC 2574 (Brinks, Walter $\&$ Kerp 2003), the data were reanalysed in a consistent manner for all sample galaxies in order to allow for a proper comparison to be made.

The integration times of the Chandra observations vary between $10 \mathrm{ks}$ and $75 \mathrm{ks}$ after removal of background flaring events. After standard calibration, point sources were identified by a wavelet algorithm, removed from the diffuse component, and analyzed independently. The resulting blanked apertures were refilled by diffuse emission from nearby regions. Spectra of the diffuse emission were fitted by two models, a Raymond--Smith (RS) and a MeKaL thermal plasma. The emission models were coupled with photoelectric absorption from a Galactic and an internal component, assuming solar metallicity for the former and the metallicity measured on HII regions for the latter.

\section{The Diffuse Emission}

Diffuse $\mathrm{X}$-ray emission which can be modelled by a hot thermal plasma was detected in all galaxies but IZw 18, VII Zw 403, and IC 2574. In those objects, 
tentative ROSAT detections of a diffuse component are identified as due to a single point source. Our $3 \sigma$ detection limits for a diffuse component over the optical bodies of the galaxies were 37,28 , and $20 \times 10^{37} \mathrm{erg} \mathrm{s}^{-1}$, for I Zw 18, VII Zw 403, and IC 2574, respectively. The $\mathrm{X}$-ray luminosities of the galaxies for which diffuse $\mathrm{X}$-ray emission was detected range from $5 \times 10^{38}$ to $2 \times 10^{41} \mathrm{erg} \mathrm{s}^{-1}$. In Fig. 1 we compare the metallicities of the galaxies (derived from spectroscopy of HII regions) with their $\mathrm{X}$-ray luminosities. Galaxies with an oxygen abundance of $12+\log (O / H) \lesssim 8.1$ were below the detection limits for diffuse hot gas. This may be an effect of the low X-ray emissivity of low-metallicity gas. However, other explanations for the non-detections cannot be excluded such as that the hot gas has already been blown out of the galaxies (see also Sect.5).

Where a hot ISM phase was detected it was found to be most intense close to the center of the galaxies and elongated toward the direction where the gradient in the neutral component of the ISM is steepest. In some cases, the X-ray emission extends beyond the optical counterparts. Diameters of the hot phase are $1-10 \mathrm{kpc}$ and temperatures are with $\sim 3 \times 10^{6} \mathrm{~K}$ very similar in all objects. The volume densities are about $0.02 \mathrm{~cm}^{-3}$ and the thermal energies $6-60 \times$ $10^{53} \mathrm{erg}$. Pressures are $P / k=1-2 \times 10^{5} \mathrm{~K} \mathrm{~cm}^{-3}$ which is about two orders of magnitude higher than the pressure of the cooler ISM. This overpressure is likely the cause for the expansion of the superwinds.

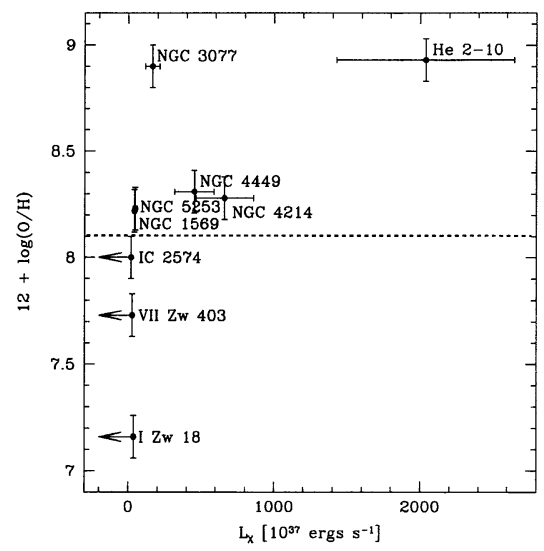

Figure 1. Oxygen abundance of the galaxies in our sample (taken from HII regions) as a function of the $\mathrm{X}$-ray luminosity of the hot gas. Note that below the dashed line hot gas was not detected.

\section{The Point Sources}

The galaxies in our sample contain up to $\sim 20$ point sources. Their spectra can be divided in to three classes: thermal plasma, power law, and black body spectra. Whereas the first class is mainly the signature of SN remnants and unresolved bubbles of hot, coronal gas, the power law spectra are most likely associated with high and low-mass $\mathrm{X}$-ray binaries and may contain some back- 
ground active galactic nuclei. The black body spectra (supersoft sources) are due to either accreting white dwarfs in the galaxies or isolated neutron stars in the Milky Way. The $\log \mathrm{N}-\log \mathrm{S}$ plot of the thermal plasma and power law spectrum sources is plotted in Fig. 2. At luminosities of $\sim 3 \times 10^{38} \mathrm{erg} \mathrm{s}^{-1}$ (somewhat larger than the Eddington limit of a $1.4 \mathrm{M}_{\odot}$ star), the slope of the plot steepens from -0.26 to -0.66 . About 8 sources in total exceed an $\mathrm{X}$-ray luminosity of $10^{39} \mathrm{erg} \mathrm{s}^{-1}$ and might therefore represent a population of ultraluminous X-ray sources which mainly appear in the most massive galaxies.

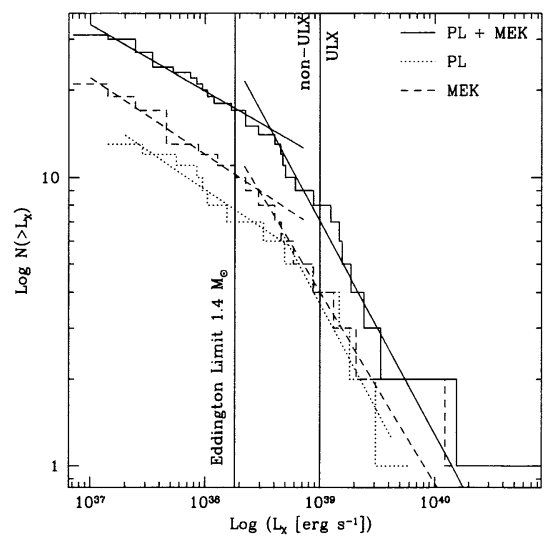

Figure 2. The number of point sources as a function of their X-ray luminosity in $\operatorname{logarithmic}$ units ( $\log N-\log S[>N]$ plot). All point sources detected in our fields were included in this graph. Two sets of sources are identified: Thermal plasma sources (dashed histogram), power law sources (dotted histogram) as well as the sum of the two (solid histogram). The lines are best fits to the histograms. In addition, the two vertical lines mark the Eddington limit for a $1.4 \mathrm{M}_{\odot}$ object and the boundary for ultraluminous $\mathrm{X}$-ray sources (defined here to exceed an X-ray luminosity of $10^{39} \mathrm{erg} \mathrm{s}^{-1}$ ).

\section{Outflow}

The overpressures of the hot gas and the morphologies mentioned above already indicate that the hot gas can at least be transported from the disks into the halos of the galaxies. A valid question is whether or not it is energetic enough to overcome the gravitational potentials of their hosts. Assuming an isothermal dark matter halo and the universal rotation curve of Persic, Salucci, \& Stel (1996) we find that the escape temperatures of the hot gas, i.e., the temperatures at which the gas kinematics exceed the escape speed of the galaxies are lower than the actually measured temperature of the hot gas. Silich \& Tenorio-Tagle (2001) calculate thresholds for the energy of a starburst at which level matter will be lost to the IGM. They include parameters such as the rotation of the host galaxy as well as the pressure of the IGM. Their thresholds are plotted in Fig. 3. 
In this figure we compare measurements of the mechanical energy stored in the superwinds on the basis of a) $\mathrm{H} \alpha$ and b) X-ray data. Taking the $\mathrm{H} \alpha$ data, only He 2-10 is not predicted to show mass loss (if non-rotating). For the energy of IZw 18 and NGC 5253 we are only able to compute lower limits which are close to the most extreme thresholds. IC 2574 will definitely not lose mass to its surroundings. This galaxy, however, is not a starburst galaxy and shows a high star formation rate within a small volume only. When applying the mechanical luminosity calculated on the basis of our X-ray data, all galaxies are predicted to lose at least some of their ISM to the IGM. About $1 \%$ of the ISM is found to be in the hot phase and this might be the maximum mass to be lost.

These calculations, however, describe only the potential for blow out on a global basis. In the case of NGC 3077 the hot gas is still stored in giant supershells (Ott, Martin, \& Walter, this volume). In addition, it appears that a massive tidal tail close to NGC 3077 (see Walter et al. 2002) blocks any gas to expand in this particular direction. A second case is NGC 4449 which is surrounded by a massive ring visible in $\mathrm{HI}$ (Hunter et al. 1998). The hot coronal gas of the starburst seems to be confined to the inner region of the ring and may cool down before substantial mass loss occurs.

This finding may have consequences for the contribution of dwarf starburst galaxies to the metal enrichment of the IGM in general. Starbursts can be triggered by galaxy-galaxy interactions. In the course of these interactions tidal tails form around the objects. On one hand, the hot gas produced by such a starburst may at least partly escape to the IGM. On the other hand, large-scale tidal tails act as barriers which are hard to overcome for the metal-enriched, hot gas.

\section{Summary and Conclusions}

- Chandra X-ray observations of eight starburst dwarf galaxies show diffuse $\mathrm{X}$-ray emission within five objects. This emission can be attributed to a hot, thermal plasma.

- The non-detection of such a component in three galaxies can be explained by their low metallicity. Other mechanisms such as blow-out, however, cannot be excluded.

- The temperature of the hot gas is with $3 \times 10^{6} \mathrm{~K}$ very similar in all objects. The thermal energies are $10^{53-54} \mathrm{erg}$ and the $\mathrm{X}$-ray luminosities are $5 \times 10^{38}$ to $2 \times 10^{41} \mathrm{erg} \mathrm{s}^{-1}$. The pressure of the hot gas is with $P / k=1-2 \times$ $10^{5} \mathrm{~K} \mathrm{~cm}^{-3}$ higher than what is usually observed in the cooler ISM. This overpressure is likely responsible for the development of the superwinds.

- X-ray point sources are found in all galaxies and show thermal plasma, power law, and black body spectra. A combined log N-log S plot steepens at luminosities larger than $\sim 3 \times 10^{38} \mathrm{erg} \mathrm{s}^{-1}$. About eight sources can be classified as ultraluminous $\mathrm{X}$-ray sources and are found in the most massive objects.

- The energetics of the hot gas is large enough to overcome the gravitational potential of the host galaxies. This may lead to partial loss of ISM (about 

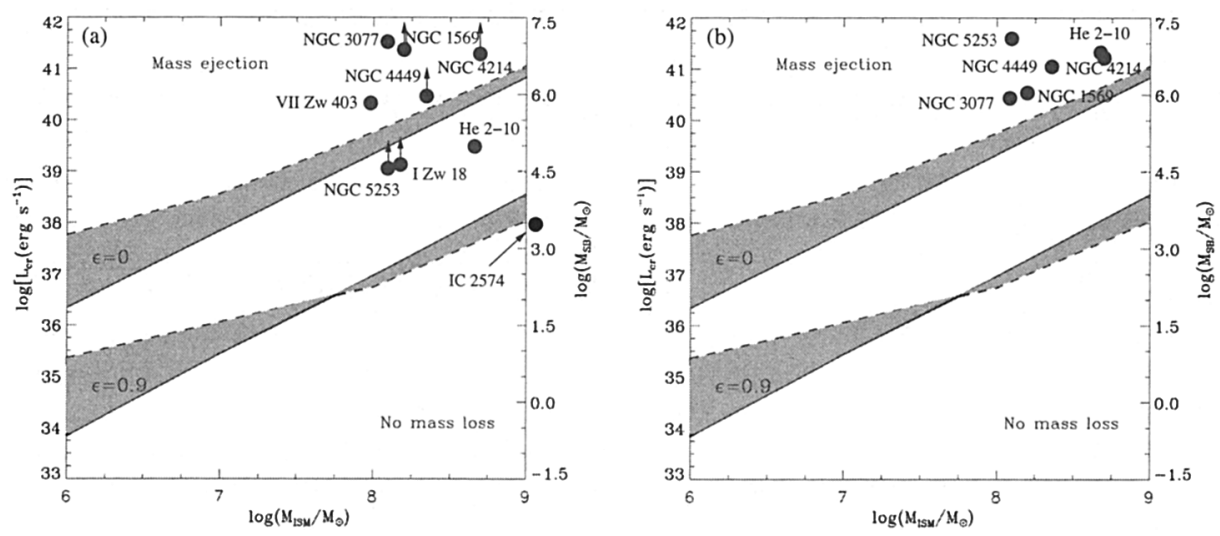

Figure 3. The mechanical luminosity of the starbursts as a function of the ISM mass of their hosts. The graph is taken from Silich \& Tenorio-Tagle (2001) with our measurements plotted on top. Thresholds for outflows were calculated for different pressures of the IGM $\left(P_{\mathrm{IGM}} / k=1-100 \mathrm{~K} \mathrm{~cm}^{-3}\right.$ grey areas $)$ and rotation speeds of the hosts ( $\varepsilon=0$ corresponds to no rotation). Outflow, i.e., mass ejection is possible if the galaxies exceed the mechanical luminosity of the threshold. The mechanical luminosities were calculated from $\mathrm{H} \alpha$ (left) and X-ray data (right). Note that for both measurements mass loss is possible for virtually all galaxies in our sample.

$1 \%$ ) to the IGM. Tidal tails when present, however, are able to reduce or even inhibit the outflow process.

\section{References}

Brinks, E., Walter, F., \& Kerp, J. 2003, Ap\&SS, 284, 627

Ferrara, A. \& Tolstoy, E. 2000, MNRAS, 313, 291

Heckman, T. M. 2002, ASP Conf. Ser. 254: Extragalactic Gas at Low Redshift, 292

Hunter, D. A., Wilcots, E. M., van Woerden, H., Gallagher, J. S., \& Kohle, S. 1998, ApJ, 495, L47

Mac Low, M. \& Ferrara, A. 1999, ApJ, 513, 142

Martin, C., Kobulnicky, H., \& Heckman, T. 2002, ApJ, 574, 663

Ott, J., Martin, C. L., Walter, F. 2003, ApJ, 594, 776

Persic, M., Salucci, P., \& Stel, F. 1996, MNRAS, 281, 27

Silich, S. \& Tenorio-Tagle, G. 2001, ApJ, 552, 91

Summers, L. K., Stevens, I. R., Strickland, D. K., Heckman, T. M. 2003, MNRAS, 342, 690

Walter, F., Weiss, A., Martin, C., \& Scoville, N. 2002, AJ, 123, 225 